\title{
Tramadol is an effective therapy for mild to severe premature ejaculation
}

$\mathrm{P}$ remature ejaculation $(\mathrm{PE})$ has an estimated prevalence of between $2 \%$ and $23 \%$, and can be associated with severe negative effects on quality of life. There is currently only one oral treatment recommended for premature ejaculationthe on-demand short-acting selective serotonin reuptake inhibitor dapoxetine. However, two studies have recently been published that have investigated the efficacy of the opioid analgesic tramadol for treating men with mild to severe PE.

A group from Colorado, USA randomized over 600 patients from 62 sites across 11 countries to receive either placebo or a tramadol orally disintegrating tablet (ODT) preparation in their doubleblind, placebo-controlled trial. The team, led by David Bar-Or, measured absolute change and fold increase in intravaginal ejaculation latency time (IELT) and also measured the change in Premature Ejaculation Profile (PEP) scores, a validated self-report questionnaire used to assess the four measures of PE as defined by the Diagnostic and Statistical Manual of Mental Disorders (DSM). The results were published in European Urology.

The study took place in two 12-week blocks, during which healthy men aged 18-65 years with a history of lifelong $\mathrm{PE}$ were randomized to receive either placebo, $62 \mathrm{mg}$ tramadol ODT or $89 \mathrm{mg}$ tramadol ODT. These doses were chosen to maximize the potential treatment effect whilst minimizing any adverse effects. The two primary end points were IELT and PEP measures, with all four PEP areas included. Any adverse effects, concomitant drug use and vital signs were also measured during the study, and each patient was used as his own control, in order to better represent the per patient efficacy of treatment.

Tramadol ODT was associated with significant increases in the median IELT in both doses tested, with a statistically significant fold increase in median IELT compared with placebo. Similar results were also seen in the subset of patients who had a baseline IELT $<1 \mathrm{~min}$, with a significant increase in absolute and fold increases in IELT seen in those treated with tramadol compared with placebo.

The PEP questionnaire was used to assess the patient's satisfaction with intercourse and that of their female partner. PEP scores were significantly increased across all four measures for the patients themselves, with the largest mean change seen in the control over ejaculation category, a result also seen in patients with a baseline IELT $<1 \mathrm{~min}$. These changes were also observed in the PEP scores for female partners of the study participants, with a significantly higher proportion of women reporting a one-category or greater improvement in their PEP score if their partner received $62 \mathrm{mg}$ or $89 \mathrm{mg}$ tramadol ODT compared with placebo.

Adverse effects were minimal, with erectile dysfunction (ED) occurring in 1\% of men and all other adverse effects having an incidence $<1 \%$.

Bar-Or summarizes: "Over 600 men were randomized in this double-blinded, multicenter, placebo-controlled study. There was a very significant improvement in IELT with the $62 \mathrm{mg}$ dose, with almost no side effects or tolerability issues. The results were even more pronounced in the men with a baseline IELT $<1$ minute (over 300 patients); in this subgroup a significant 2.4-fold increase in IELT was observed with $62 \mathrm{mg}$ tramadol ODT".

The conclusions drawn by Bar-Or and colleagues are also reflected in a similar study recently published in Zhonghua Nan Ke Xue. In this study, Xiong et al. investigated the safety and efficacy of tramadol hydrochloride in combination with behavioral modification. Patients were randomized to receive either behavioral therapy alone or with $50 \mathrm{mg}$ tramadol hydrochloride approximately

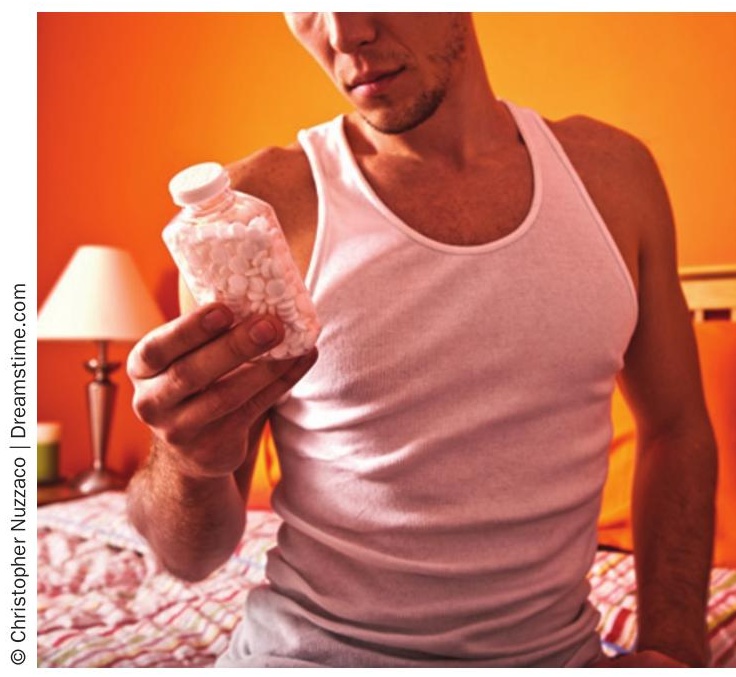

$2 \mathrm{~h}$ before sexual activity, for 8 weeks. Interestingly, both groups of patients experienced an improvement in IELT compared with pretreatment times, with a $72 \%$ improvement seen in the tramadol and behavioral therapy group, and $47 \%$ in the group who received behavioral therapy alone.

These data suggest that tramadol, in either its ODT or hydrochloride form, could provide an effective and safe treatment for men with mild to severe PE. Bar-Or and colleagues are now pursuing the possibility of a combined treatment, including tramadol ODT with a phosphodiesterase type 5 inhibitor, in order to treat both disorders and to combat the adverse effect of tramadol ODT on erectile function, and vice versa.

\section{Annette Fenner}

\footnotetext{
Original articles Bar-Or, D. et al. A randomized double-blind, placebo-controlled multicenter study to evaluate the efficacy and safety of two doses of the Tramadol orally disintegrating tablet for the treatment of premature ejaculation within less than 2 minutes. Eur. Urol. doi:10.1016/j. eururo.2011.08.039 | Xiong, G. G. et al. Safety and efficacy of tramadol hydrochloride with behavioral modification in the treatment of premature ejaculation [Chinese]. Zhonghua Nan Ke Xue 17, 538-541 (2011)
} 